\title{
Microestrutura e propriedades magnéticas da hexaferrita de bário dopada com cromo e sinterizada por micro-ondas
}

\section{(Microstructure and magnetic properties of microwave-sintered chromium-doped barium hexaferrite)}

\author{
W. S. Castro ${ }^{1,2}$, L. J. D. Costa ${ }^{3}$, R. R. Corrêa ${ }^{2}$, A. J. A. de Oliveira ${ }^{2}$, R. H. G. A. Kiminami ${ }^{1}$ \\ ${ }^{1}$ Departamento de Engenharia de Materiais, Universidade Federal de S. Carlos, Rod. Washington Luiz, km 235, \\ C. P. 676, S. Carlos, SP, Brasil 13565-905 \\ ${ }^{2}$ Departamento de Eletroeletrônica, Instituto Federal de Educação, Ciência e Tecnologia do Maranhão, \\ Av. Getúlio Vargas 4, Monte Castelo, S. Luís, MA, Brasil 65030-005 \\ ${ }^{3}$ Departamento de Física, Universidade Federal de S. Carlos, Rod. Washington Luiz, km 235, C. P. 676, \\ S. Carlos, SP Brasil 13565-905
}

\begin{abstract}
Resumo
Este trabalho teve por objetivo sinterizar cerâmicas de hexaferrita de bário comercial em forno de micro-ondas, visando obter propriedades magnéticas tão boas quanto aquelas obtidas por sinterização convencional. Amostras de hexaferrita de bário foram dopadas com cromo, prensadas isostaticamente e sinterizadas em forno de micro-ondas e convencional. A caracterização foi por difração de raios X, microscopia eletrônica de varredura, determinação de densidade aparente e curvas de histerese magnética. $\mathrm{O}$ tempo de queima da hexaferrita de bário por micro-ondas foi em menos de $20 \%$ do tempo gasto da queima convencional. As amostras apresentaram tamanho de grão um pouco inferior, densidade e magnetização de saturação semelhante às obtidas pela sinterização convencional, mostrando o alto potencial de aplicação da técnica de micro-ondas para o processamento da hexaferrita de bário comercial. A adição de óxido de cromo nas amostras de hexaferrita de bário resultou na melhoria das propriedades magnéticas.

Palavras-chave: sinterização, micro-ondas, hexaferrita de bário, cromo.
\end{abstract}

\begin{abstract}
The purpose of this work was to sinter commercial barium hexaferrite ceramic in a microwave oven, aiming to obtain magnetic properties similar to those obtained by conventional sintering. Samples of barium hexaferrite were doped with chromium, isostatically pressed and sintered in a microwave oven and a conventional furnace for comparison. The sintered samples were characterized by $X$-ray diffraction, scanning electron microscopy, bulk density, and magnetic hysteresis curves. Microwave sintering of the barium hexaferrite took less than $20 \%$ of the time spent in conventional sintering. The microwave sintered samples showed a somewhat smaller grain size, but their density and saturation magnetization were similar to those obtained by conventional sintering, indicating the promising potential of processing commercial barium hexaferrite by microwaves. The addition of chromium oxide to the barium hexaferrite samples improved their magnetic properties.
\end{abstract}

Keywords: sintering, microwaves, barium hexaferrite, chromium.

\section{INTRODUÇÃO}

As hexaferritas de bário têm tido e continuam tendo um papel importante no desenvolvimento das cerâmicas magnéticas. São usadas principalmente como magneto permanente [1], mas a cada melhoria feita em suas propriedades, as suas aplicações são ampliadas para as mais diversas áreas tecnológicas, como em sistemas de armazenamento de dados, biossensores, dispositivos eletroeletrônicos, diagnósticos médicos, e outras. As propriedades magnéticas das hexaferritas dependem da sua microestrutura, que são modificadas por variações no processamento, pelo tratamento térmico e pela introdução de dopantes [2]. Os dopantes modificam as propriedades magnéticas, pela substituição dos íons $\mathrm{Fe}^{3+}$ por íons bi, tri e tetravalentes, ou combinação dos mesmos, visando a ocupação de sítios spin down, para consequentemente aumentar a magnetização da rede [3]. Nesse sentido, muito interesse tem sido devotado à análise da influência de dopantes na melhoria das propriedades magnéticas das hexaferritas [4-6]. Para diversos autores, as propriedades magnéticas da hexaferrita de bário estão intimamente relacionadas com a distribuição dos íons dopantes nos cinco sítios cristalográficos de sua rede cristalina [7-14]; assim sendo, a substituição de íons $\mathrm{Fe}^{3+}(5 \mu \mathrm{B})$ por íons $\mathrm{Cr}^{3+}(3 \mu \mathrm{B})$ na hexaferrita de bário, origina mudanças significativas nas propriedades de magnetização de saturação (Ms), remanência $(\mathrm{Mr})$ e coercividade $(\mathrm{Hc})$. A magnetização de saturação, como uma propriedade intrínseca, é determinada pela composição dos materiais, enquanto a coercividade, 
como uma propriedade extrínseca, é determinada pela microestrutura, que é muito influenciada pelos métodos de processamento. A microestrutura é dependente do tamanho de partícula, homogeneidade do material precursor e das condições de sinterização. Logo, para se controlar o desenvolvimento microestrutural e se atingir as propriedades desejadas no produto final, parâmetros como a temperatura, tempo de sinterização, e taxa de aquecimento devem ser controlados e otimizados [15].

A sinterização por micro-ondas tem sido reportada como benéfica na obtenção de microestruturas refinadas (com pequeno tamanho de grão) e elevadas densificações nos corpos cerâmicos em um curto tempo. Isto porque a sinterização por micro-ondas baseia-se na transformação da energia de micro-ondas em calor dentro do material, o que resulta em significante economia de energia, redução do tempo de processamento e melhoria na uniformidade microestrutural dos corpos cerâmicos [16-21], o que vem mostrando ser o fator decisivo na aceitação do uso das micro-ondas na sinterização de materiais cerâmicos.

As cerâmicas são em geral transparentes às micro-ondas, mas quando aquecidas acima de determinada temperatura (temperatura crítica), passam a absorver mais eficientemente essa radiação. A sinterização híbrida combina o aquecimento por micro-ondas com o aquecimento convencional simultaneamente. É uma técnica que produz aquecimento rápido em baixas temperaturas e uniformidade na distribuição da temperatura nas amostras durante a sinterização $[15$, 16]. Durante o aquecimento inicial, utiliza-se um susceptor colocado perto da amostra para fazê-la alcançar a temperatura crítica mais rapidamente, quando então passa a interagir com as micro-ondas, até alcançar a temperatura desejada. Vários trabalhos vêm abordando a sinterização por micro-ondas de materiais cerâmicos, como a alumina, que é transparente às micro-ondas [22, 23], observando-se excelentes resultados em grande parte desses estudos. Entretanto, são poucos os trabalhos abordando a queima por micro-ondas da hexaferritas de bário e, menores ainda, estudos envolvendo a influência de dopantes no desenvolvimento microestrutural da hexaferrita de bário quando submetida à sinterização por micro-ondas. Assim, este trabalho tem como objetivo avaliar o efeito da sinterização por micro-ondas da hexaferrita de bário com e sem dopante de cromo na microestrutura e nas propriedades magnéticas.

\section{MATERIAIS E MÉTODOS}

Foram utilizados pó de hexaferrita de bário comercial (Supergauss) e óxido de cromo (Vetec). A hexaferrita de bário sem dopante $(\mathrm{BaM})$ e as dopadas com $\mathrm{Cr}_{2} \mathrm{O}_{3}$ (BaMCr's) foram dispersas em meio alcoólico por $12 \mathrm{~h} \mathrm{em}$ moinho de bolas. Foram adicionadas pequenas quantidades de $\mathrm{Cr}_{2} \mathrm{O}_{3}$ na hexaferrita de bário, seguindo a composição química $\mathrm{BaCrxFe} 12-\mathrm{xO} 19$, de modo a se ter 0,2(BaMCr2), $0,3(\mathrm{BaMCr} 3)$ e $0,4(\mathrm{BaMCr} 4) \mathrm{mol} \%$ de $\mathrm{Cr}$ no material final. Os corpos-de-prova (diâmetro $10 \mathrm{~mm}$ e espessura 2,5 mm) foram confeccionados por prensagem uniaxial seguida por prensagem isostática a $200 \mathrm{MPa}$ em prensa AIP CP360. A densidade a verde das amostras situou-se em torno $60 \%$. As amostras foram sinterizadas a $1280{ }^{\circ} \mathrm{C}$ em forno convencional (Lindberg/Blue M) com taxa de aquecimento $10{ }^{\circ} \mathrm{C} /$ min e patamar de sinterização $60 \mathrm{~min}$, e por microondas a $1280^{\circ} \mathrm{C} \mathrm{em}$ forno de micro-ondas (Cober MS6K) com taxa de aquecimento $50^{\circ} \mathrm{C} / \mathrm{min}$ e patamar de sinterização 30 min. A densidade das amostras sinterizadas foi determinada pelo método de Arquimedes com imersão em água. Foram executados ensaios de difração de raios X (Siemens 5100, com radiação $\mathrm{Cuk} \alpha$ ) nas amostras sinterizadas para avaliar as fases relacionadas à presença de cromo. As amostras foram analisadas por microscopia eletrônica de varredura (Philips XL30-FEG), em superfícies polidas e fraturadas. O tamanho médio dos grãos foi calculado a partir das medidas dos grãos nas micrografias das amostras, com os programas Image J e OringinPro 8. Para a determinação das propriedades magnéticas foram feitas medidas de magnetização em função do campo magnético aplicado, em um magnetômetro de amostra vibrante (VSM Controller 4500 EG\&G), instalado em um eletroímã com campo máximo de $8 \mathrm{kOe}$, à temperatura ambiente.

\section{RESULTADOS E DISCUSSÃO}

As medidas de densidade relativa dos corpos da hexaferrita de bário sem e com adição de óxido de cromo sinterizadas em forno convencional (SC) e de micro-ondas (SM) a $1280^{\circ} \mathrm{C}$ são apresentadas na Tabela I.

Tabela I - Densidade relativa das amostras sinterizadas em forno convencional (SC) e por micro-ondas (SM).

[Table I - Relative density of conventionally (SC) and microwave (SM) sintered samples.]

\begin{tabular}{ccc}
\hline Amostras & DR (\%) - SC & DR (\%) - SM \\
\hline BaM & 94,8 & 94,3 \\
BaMCr2 & 93,9 & 92,6 \\
BaMCr3 & 92,6 & 92,2 \\
BaMCr4 & 92,3 & 91,5 \\
\hline
\end{tabular}

As densidades relativas das amostras de hexaferrita de bário sem dopante, sinterizadas em forno convencional (SC) e de micro-ondas (SM) foram similares. As amostras dopadas com cromo também tiveram suas densidades bem próximas nas duas sinterizações. A adição de cromo tendeu a reduzir a densificação de hexaferrita de bário em ambos os processos de sinterização, e quanto maior foi o teor, menor a densidade.

Os difratogramas de raios $\mathrm{X}$ das amostras com e sem cromo sinterizados convencionalmente e por micro-ondas são apresentados na Fig. 1, na qual se observam picos relativos à fase magnetoplumbita em todos os casos, sem nenhuma fase secundária.

As micrografias de hexaferrita de bário sem e com adição de cromo e sinterizadas em forno de convencional (SC) a 

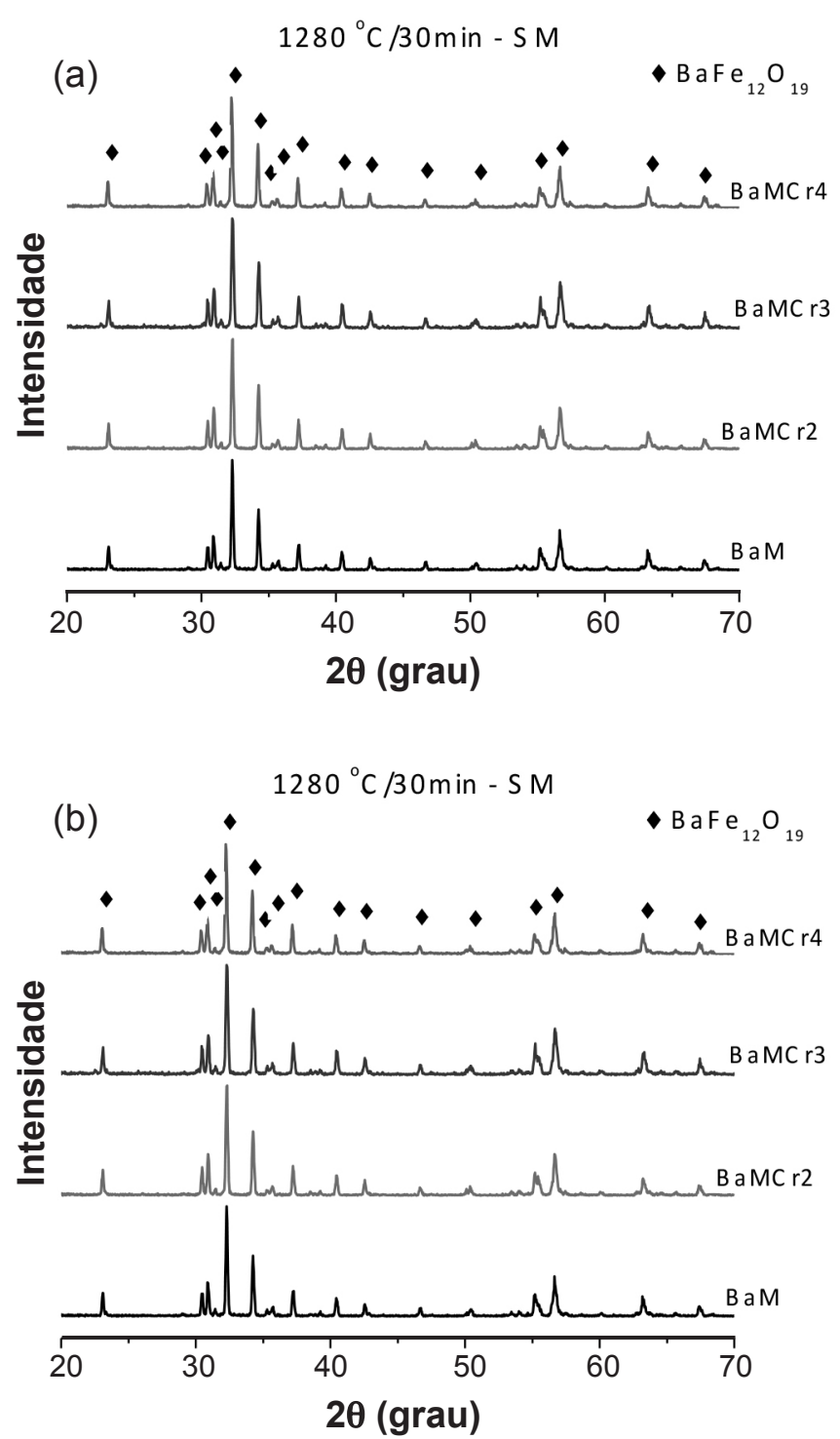

Figura 1: Difratogramas de raios X das amostras de hexaferrita de bário sem e com dopante, sinterizadas em forno convencional (SC) e de micro-ondas (SM).

[Figure 1: XRD patterns of conventionally (SC) and microwave (SM) sintered barium hexaferrite samples.]

$1280^{\circ} \mathrm{C} / 1 \mathrm{~h}$ e por micro-ondas (SM) a $1280^{\circ} \mathrm{C} / 30$ min, estão apresentadas na Fig. 2.

A adição de cromo favoreceu o crescimento dos grãos e redução da densificação. Observa-se de um modo geral uma microestrutura bem heterogênea com grãos de morfologia similar, tanto nas amostras sinterizadas convencionalmente, que foram polidas e atacadas termicamente, quanto nas amostras sinterizadas por micro-ondas, que foram fraturadas e tratadas quimicamente, evitando-se assim o tratamento térmico e eventual alteração de sua morfologia. Os valores de tamanho médio de grãos das amostras sinterizadas em forno convencional (SC) a $1280{ }^{\circ} \mathrm{C} / 1 \mathrm{~h}$ e em forno de microondas a $1280^{\circ} \mathrm{C} / 30 \mathrm{~min}$, calculados pelos programas Image J e o OringePro 8 estão apresentados na Tabela II.

Os tamanhos médios dos grãos das amostras sinterizadas por micro-ondas foram inferiores aos obtidos pela sinterização convencional. Isto está relacionado à forma de aquecimento na sinterização por micro-ondas (volumétrico) e ao menor tempo de exposição da amostra em alta temperatura, devido a uma maior taxa de aquecimento e menor tempo de patamar de sinterização, o que produziu menor crescimento dos grãos. Além disso, a adição de cromo quase não alterou o tamanho médio de grão das amostras sinterizadas convencionalmente, mantendo-os praticamente constantes, enquanto que na sinterização por micro-ondas houve uma maior variação do tamanho médio de grão, e ainda, quanto maior foi o teor de cromo nas amostras, maior os tamanhos médios dos grãos, mas mantendo-se sempre inferiores aos tamanhos médios de grãos das amostras sinterizadas em forno convencional.

As curvas de magnetização em função do campo magnético aplicado $(\mathrm{MxH})$ das amostras de hexaferrita de bário com e sem dopante, sinterizadas em forno convencional e por micro-ondas a $1280{ }^{\circ} \mathrm{C}$ são apresentadas na Fig. 3, na qual se observa que as curvas $\mathrm{MxH}$, apresentam um largo ciclo de histerese, característico das ferritas duras que possuem elevado valor de coercividade, e a não saturação das amostras para o campo magnético externo aplicado.

A Tabela III mostra os parâmetros magnéticos (magnetização de saturação, magnetização remanente e coercividade) obtidos a partir das curvas $\mathrm{M} \mathrm{x} \mathrm{H}$.

Para a hexaferrita de bário sem dopagem, sinterizada em forno convencional e por micro-ondas (BaM-SC e BaM$\mathrm{SM}$ ), os ciclos de histerese são bastante similares, e como apresentaram praticamente densidades similares, a redução do tamanho médio de grãos da hexaferrita sinterizada por micro-ondas influenciou no aumento da coercividade com uma pequena diminuição da magnetização de saturação e remanência. Para as amostras sinterizadas convencionalmente, a adição de cromo ocasionou uma diminuição da magnetização de saturação e aumento da coercividade. A diminuição da magnetização de saturação pode ser também influenciada por fatores extrinsicos como a microestrutura e densidade das ferritas [24]. Neste caso, a diminuição da densidade observada, ou o aumento da porosidade compromete a contribuição rotacional dos spins, uma vez que os poros atuam como centros de aprisionamento dos spins dos elétrons. Já a coercividade Hc aumentou quase que linearmente com o aumento do teor de cromo nas composições, o que pode estar relacionado ao aumento do campo anisotrópico magnetocristalino [24]. Para as amostras sinterizadas por micro-ondas, a adição de cromo ocasionou o aumento da magnetização de saturação, remanência e coercividade. Isto ocorreu porque a dopagem resultou também no aumento do tamanho médio de grãos, que fez diminuir a área de contorno de grãos, facilitando o movimento das paredes de domínio, e aumentando a magnetização de saturação. Pode estar relacionado também a uma maior ocupação dos íons $\mathrm{Cr}^{3+}$ nos sítios $\mathrm{Fe}^{3+}$ de spin down (4f2) da estrutura hexaferrita, resultando no aumento da magnetização da rede e consequentemente da magnetização de saturação [13]. A amostra de hexaferrita dopada com $4 \mathrm{~mol} \%$ de cromo (BaMCr4-SM), obteve propriedades magnéticas superiores aos da hexaferrita sem dopagem, 
(a)

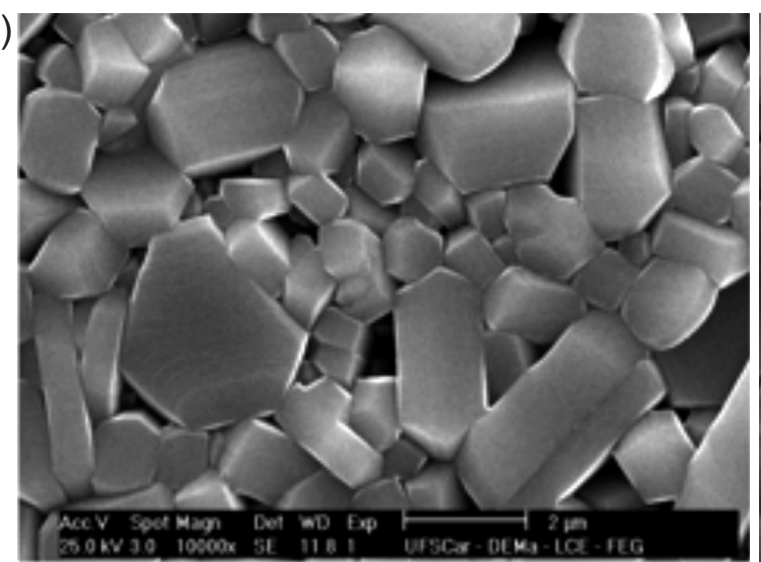

(c)

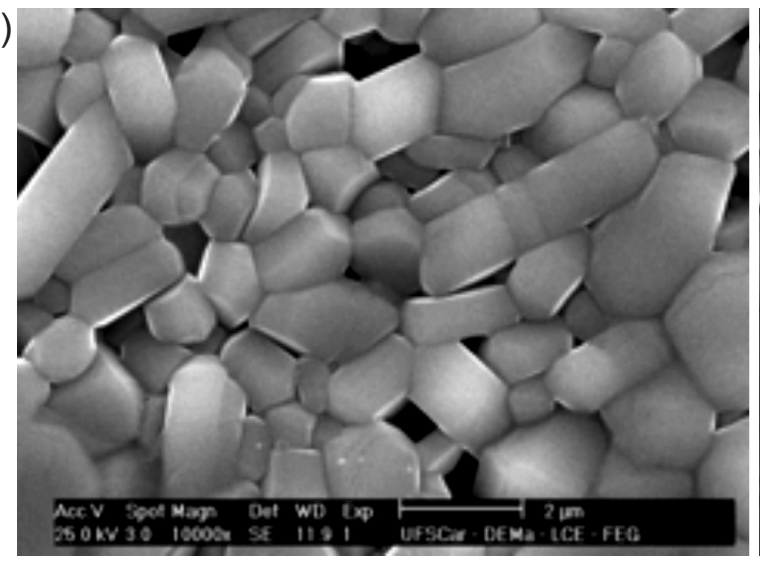

(e)

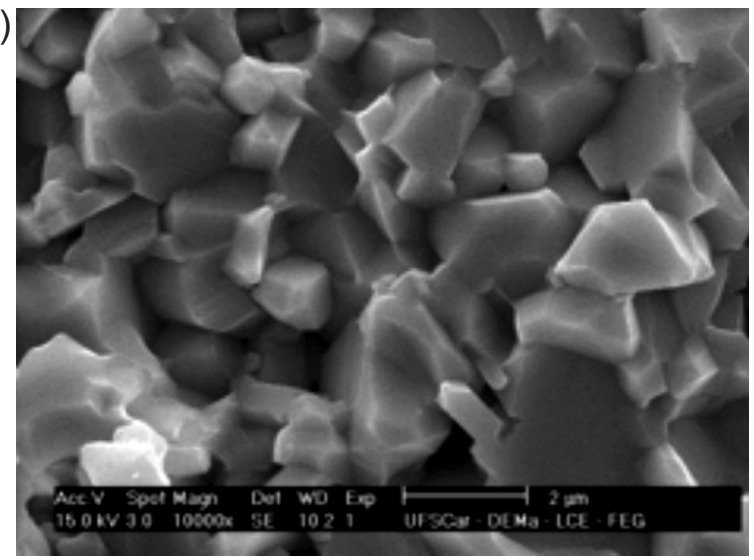

(g)

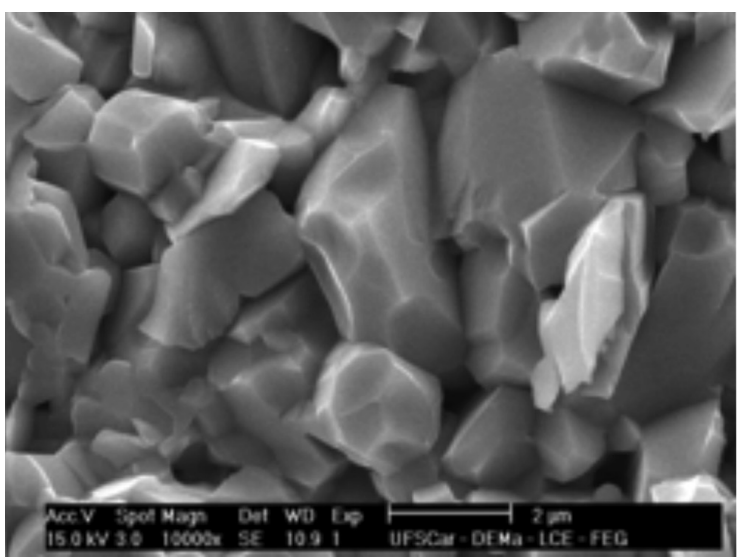

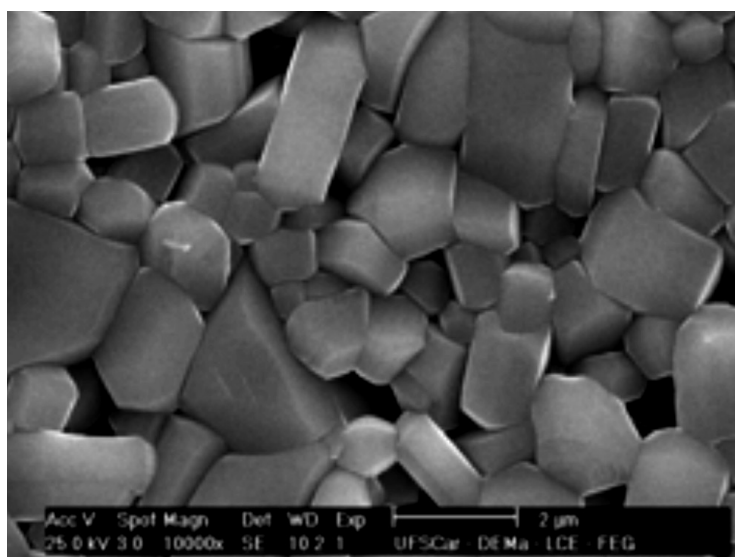

(b)

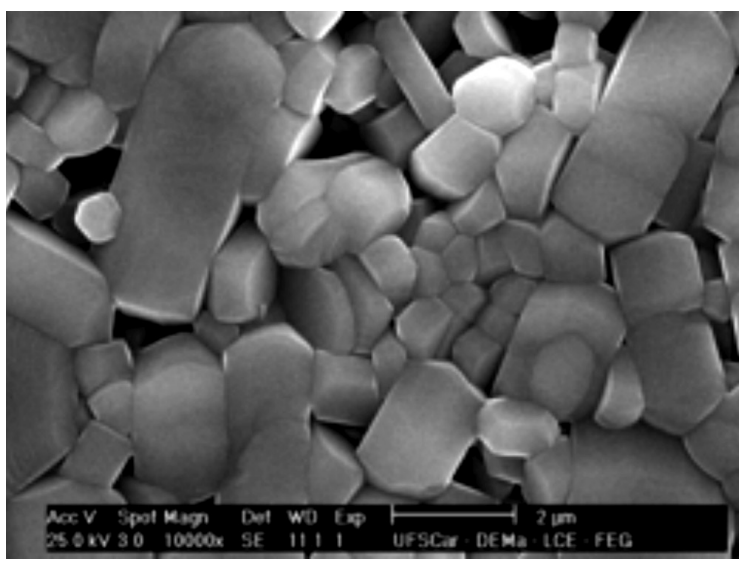

(d)

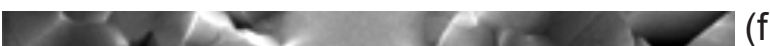

(f)
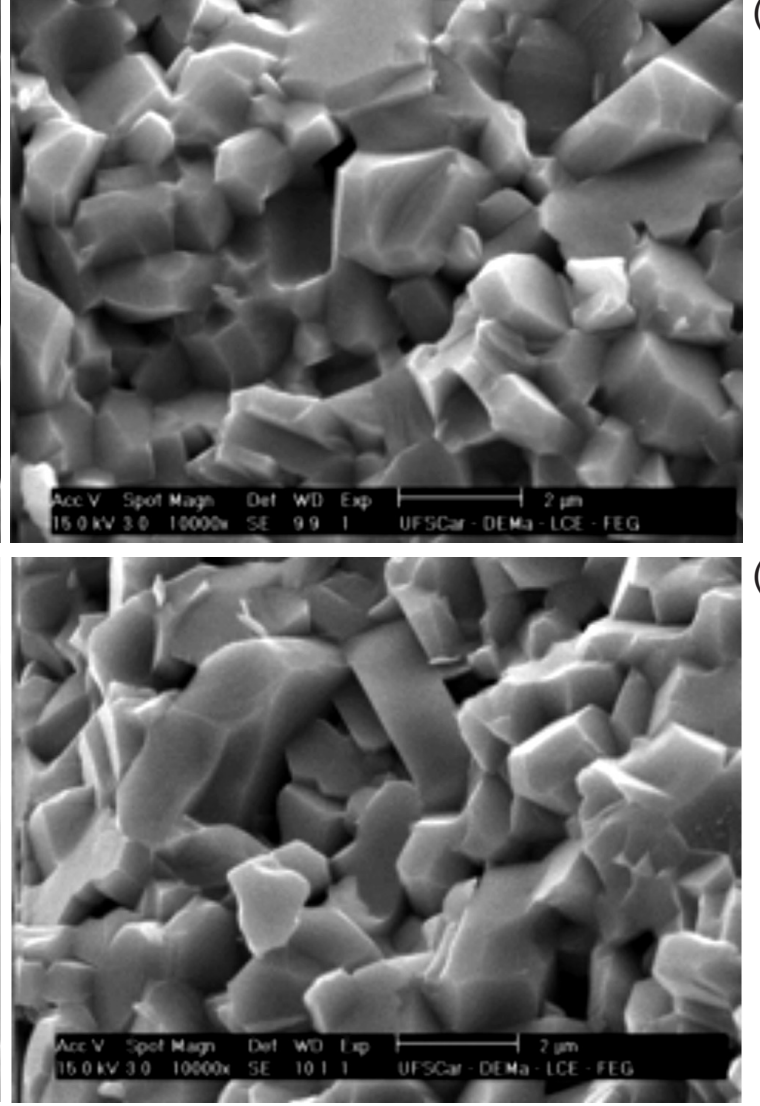

(h)

Figura 2: Micrografias obtidas em microscópio eletrônico de varredura de hexaferrita de bário, sem e com dopante, sinterizadas em forno convencional (a, b, c, d) e de micro-ondas (e, f, g, h).

[Figure 2: SEM micrographs of conventionally $(a, b, c, d)$ and microwave $(e, f, g, h)$ sintered barium hexaferrite samples.] 
Tabela II - Tamanho médio de grãos das amostras sinterizadas em forno convencional (SC) e por micro-ondas (SM).

[Table II - Average grain size of conventionally (SC) and microwave (SM) sintered samples.]

\begin{tabular}{ccc}
\hline Amostra & $\begin{array}{c}\text { Tamanho médio } \\
\text { de grão }(\mu \mathrm{m})-\mathrm{SC}\end{array}$ & $\begin{array}{c}\text { Tamanho médio } \\
\text { de grão }(\mu \mathrm{m})-\mathrm{SM}\end{array}$ \\
\hline BaM & $1,79 \pm 0,6$ & $1,62 \pm 0,5$ \\
BaMCr2 & $1,78 \pm 0,6$ & $1,69 \pm 0,6$ \\
BaMCr3 & $1,79 \pm 0,7$ & $1,75 \pm 0,6$ \\
BaMCr4 & $1,81 \pm 0,7$ & $1,74 \pm 0,7$ \\
\hline
\end{tabular}
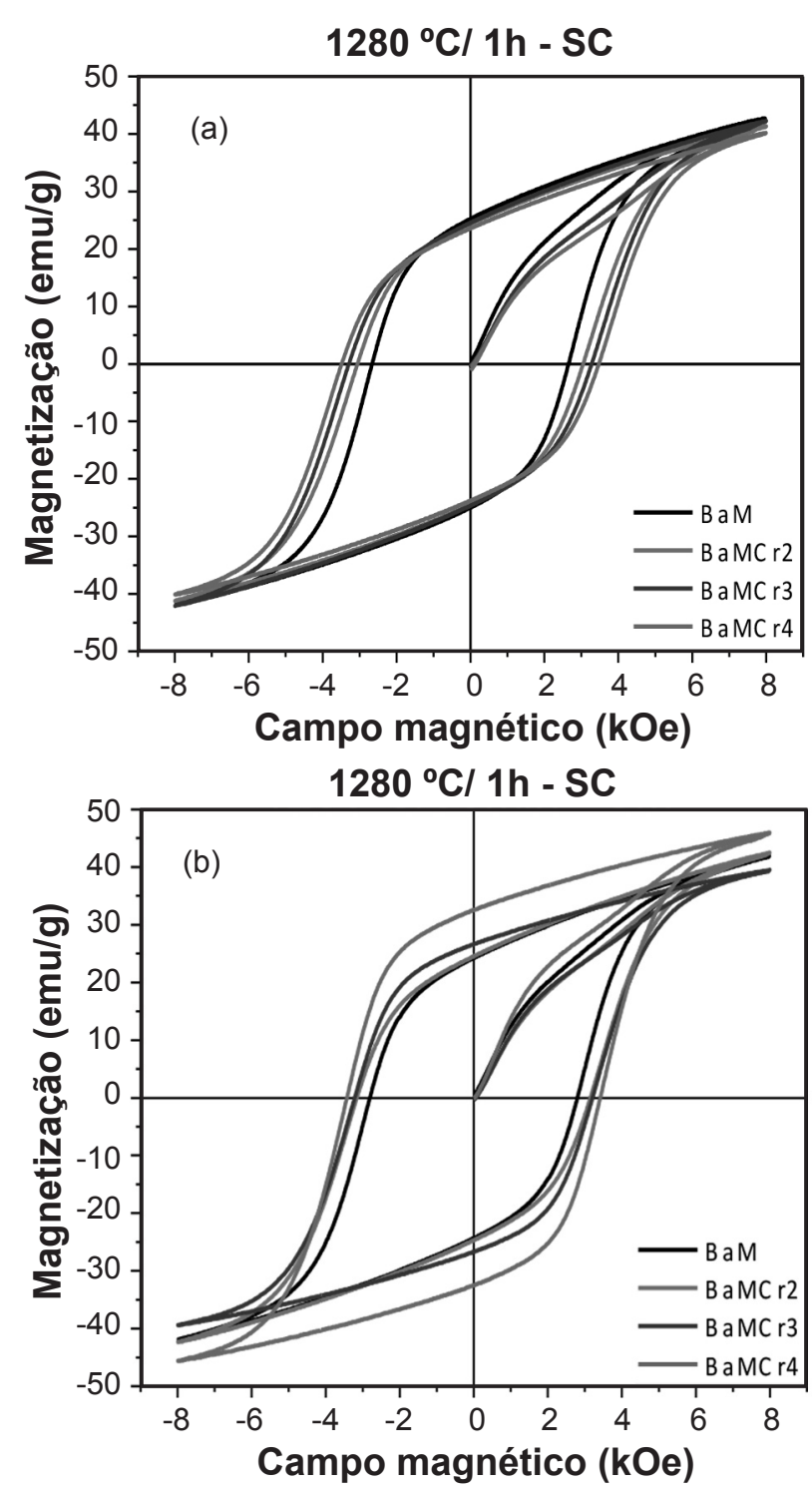

Figura 3: Curvas de magnetização em função do campo magnético aplicado das amostras de hexaferritas de bário, sinterizadas em forno convencional (SC) e de micro-ondas (SM).

[Figure 3: Curves of magnetization as a function of the applied magnetic field in conventional (SC) and microwave (SM) sintered barium hexaferrite samples.]

tanto as sinterizadas em forno convencional quanto por micro-ondas, visto que este teor de dopante na BaMCr4-SM resultou no aumento da magnetização de saturação, conforme
Tabela III - Parâmetros magnéticos das amostras de hexaferritas de bário, sem e com dopante, sinterizadas em forno convencional (SC) e por micro-ondas (SM).

[Table III - Magnetic parameters of conventionally (SC) and microwave (SM) sintered barium hexaferrite samples.]

\begin{tabular}{ccccccc}
\hline & \multicolumn{2}{c}{ Ms $(\mathrm{emu} / \mathrm{g})$} & \multicolumn{2}{c}{$\mathrm{Mr}(\mathrm{emu} / \mathrm{g})$} & \multicolumn{2}{c}{ Hc $(\mathrm{kOe})$} \\
Amostras & SC & SM & SC & SM & SC & SM \\
\hline BaM & 42,56 & 42,04 & 25,2 & 24,3 & 2,65 & 2,80 \\
BAMCr2 & 41,31 & 42,48 & 24,39 & 24,56 & 3,06 & 3,16 \\
BAMCr3 & 42,25 & 40,00 & 24,58 & 26,63 & 3,28 & 3,29 \\
BAMCr4 & 40,18 & 45,95 & 23,57 & 32,59 & 3,47 & 3,42 \\
\hline
\end{tabular}

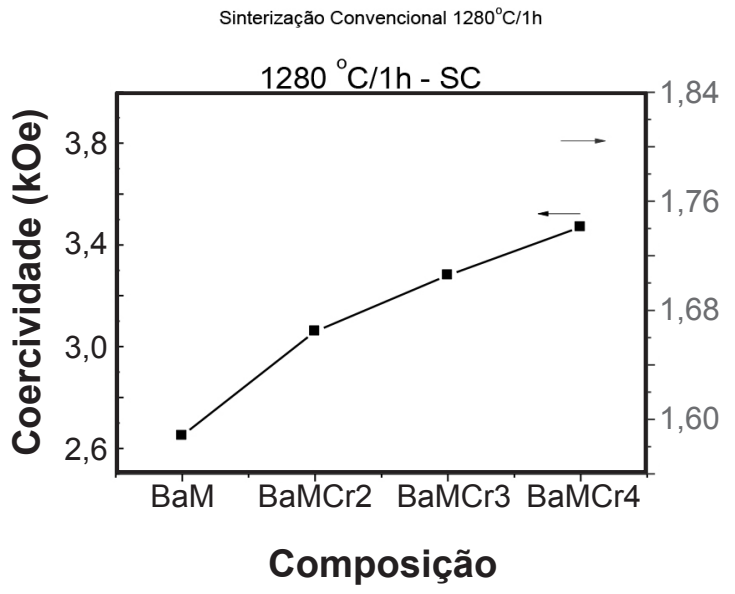

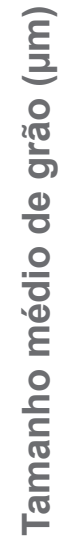

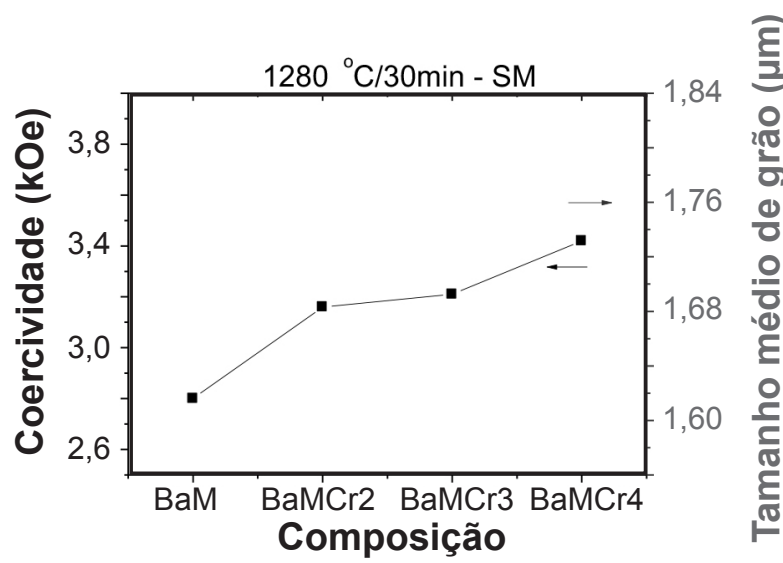

Figura 4: Coercividade e tamanho médio de grão em função da adição de cromo nas amostras sinterizadas em forno convencional (SC) e de micro-ondas (SM) a $1280^{\circ} \mathrm{C}$.

[Figure 4: Coercivity and average grain size of samples sintered at $1280{ }^{\circ} \mathrm{C}$ conventionally (SC) and by microwaves (SM) as a function of chromium content.]

descrição anterior. Na amostra BaMCr4-SM, a adição de cromo resultou também na diminuição do tamanho médio de grão, com aumento da coercividade, mas ficou inferior à coercividade obtida pela amostra BaMCr4-SC. Isto pode ser atribuído ao elevado ganho da magnetização de saturação da amostra, que ocasionou a diminuição da coercividade [25], conforme previsto pela equação: 


$$
\mathrm{Hc}=\alpha(2 \mathrm{k} / \mathrm{Ms})
$$

na qual $\alpha$ é uma constante de proporcionalidade, $\mathrm{k}$ a anisotropia magnética, e Ms a magnetização de saturação.

A Fig. 4 apresenta o comportamento do tamanho médio de grãos e coercividade em função da adição de cromo na hexaferrita de bário, sinterizadas em forno convencional e de micro-ondas a $1280^{\circ} \mathrm{C}$.

$\mathrm{O}$ aumento do teor de cromo na hexaferrita de bário para as amostras sinterizadas convencionalmente fez aumentar a coercividade, mantendo o tamanho médio de grãos quase constante, ou seja, a adição de cromo ocasionou diminuição da densidade, mas não exerceu uma influência significativa no crescimento dos grãos. Já na sinterização por micro-ondas, o aumento do teor de cromo nas amostras também fez aumentar a coercividade sendo que para apenas a amostra de hexaferrita dopada com $0,4 \%$ de cromo observou-se que houve uma diminuição no tamanho médio de grãos, resultando numa elevação ainda maior da coercividade (maior inclinação). Os valores de tamanho médio de grãos na sinterização por microondas foram inferiores aos da sinterização convencional, o que pode ser atribuído, ao tipo de sinterização e ao menor tempo de exposição das amostras em altas temperaturas, para atingir densidades similares.

\section{CONCLUSÕES}

A sinterização por micro-ondas foi favorável para a obtenção de hexaferritas de bário densas e com tamanhos de grãos inferiores às sinterizadas em forno convencional. As composições estudadas apresentaram a fase magnetoplumbita, sem fases secundárias. O tempo de queima das amostras sinterizadas por micro-ondas foi de aproximadamente $20 \%$ do tempo gasto na sinterização convencional. A adição de $0,4 \%$ de óxido de cromo interferiu diretamente na microestrutura causando aumento na magnetização e na coercividade, permitindo o uso desses materiais, sinterizados por microondas, como magnetos duros com ciclos de magnetização relativamente largos.

\section{AGRADECIMENTOS}

À FAPESP pelo apoio financeiro (Procs. 2008/04025-0 e 2012/24025-0), à CAPES pela bolsa concedida, ao programa de Pós-graduação em Ciência e Engenharia de Materiais da Universidade Federal de S. Carlos, e à Supergauss pela doação da hexaferrita de bário.

\section{REFERÊNCIAS}

[1] V. V. Soman, V. M. Nanoti, D. K. Kulkarni, Ceram. Int.
39 (2013) 5713-5723.

[2] V. J. Silva, A. P. A. Diniz, P. T. A Santos, D. A. Vieira, A. C. F. M. Costa, D. R. Cornejo, Rev. Elet. Mat. Proc. 1, 2 (2006) 09-17.

[3] J. F. Wang, C. B. Ponton, I. R. Harris, J. Mag. Mag. Mat. 234 (2001) 233-240.

[4] S. Chang, S. Kangning, C. Pengfei, J. Mag. Mag. Mat. 324 (2012) 802-805.

[5] S. Jauhar, J. Singh, K. Chandra, S. Bansal, S. Singhal, Powder Tech. 212 (2011) 802-805.

[6] C. S. Kim, S. Y. On, J. H. Son, IEEE Trans. Mag. 35 (1999) 3160-3162.

[7] M. N. Ashiq, M. J. Iqbal, I. H. Gul, J. Mag. Mag. Mat. 323 (2011) 259-263.

[8] A. M. Gadalla, H. E. Shutz, H. W. Hennicke, J. Mag. Mag. Mat. 1 (1976) 241-250.

[9] V. N. Dhage, M. L. Mane, M. K. Babrekar, C. M. Kale, K. M. Jadhav, J. Alloys Compnd. 509 (2011) 4394-4398.

[10] S. Ounnunkad, P. Winotai, J. Mag. Mag. Mat. 301 (2006) 292-300.

[11] C. Kim, A. Sung, Y. Sung, J. H. Son. IEEE Trans. Mag. 35 (1999) 3160-3162.

[12] J. Qiu, M. Gu, H. Shen, J. Mag. Mag. Mat. 295, 3 (2005) 263-268.

[13] A. Ihsan, M. U. Islam, M. S. Awan, M. Ahmad, J. Alloys Compnd. 547 (2013) 118-125.

[14] Q. Fang, H. Cheng, K. Huang, J. Wang, Y. Jiao, J. Mag. Mag. Mat. 294, 3 (2005) 281-286.

[15] R. R. Menezes, R. H. G. A. Kiminami, J. Mater. Proc. Tech. 203 (2008) 513-517.

[16] R. R. Menezes, P. M. Souto, R. H. G. A. Kiminami, Cerâmica 53, 325 (2007) 1-10.

[17] A. C. F. M. Costa, M. R. Morelli, R. H. G. A. Kiminami, Cerâmica 49, 311 (2003) 168-173.

[18] M. Sorescu, L. Diamandescu, R. Peelamedu, R. Roy, P. Yadoji, J. Mag. Mag. Mat. 279 (2004) 195-201.

[19] S. Das, K. Mukhopadhyay, S. Datta, D. Basu, Bull. Mater. Sci. 32 (2009) 1-13.

[20] R. R. Menezes, P. M. Souto, R. H. G. A. Kiminami, Cerâmica 53, 326 (2007) 108-115.

[21] R. R. Menezes, P. M. Souto, R. H. G. A. Kiminami, J. Mater. Proc. Tech. 190 (2007) 223-229.

[22] M. Oghbaei, O. Mirzaee, J. Alloys Compnd. 494 (2010) 175-189.

[23] R. R. Menezes, P. M. Souto, R. H. G. A. Kiminami, Cerâmica 53, 327 (2007) 218-226.

[24] M. A. Gabal, Y. M. Angari, Mater. Chem. Phys. 118 (2009) 153-160.

[25] A. A. Nourbakhsh, M. Nourbakhsh, M. Nourbakhsh, M. Shaygan, K. J. D. Mackenzie, J. Mater. Sci: Mater Eletr. 22 (2011) 1297-1302.

(Rec. 11/07/2014, Ac. 10/10/2014) 